\title{
Methylation of bone morphogenetic protein 2 is associated with poor prognosis in colorectal cancer
}

\author{
TOMIYUKI MIURA ${ }^{1}$, MEGUMI ISHIGURO ${ }^{2}$, TOSHIAKI ISHIKAWA ${ }^{3}$, \\ SATOSHI OKAZAKI ${ }^{3}$, HIRONOBU BABA ${ }^{1}$, AKIFUMI KIKUCHI ${ }^{1}$, SHINICHI YAMAUCHI ${ }^{1}$, \\ TAKATOSHI MATSUYAMA ${ }^{1}$, HIROYUKI UETAKE ${ }^{3}$ and YUSUKE KINUGASA ${ }^{1}$ \\ Departments of ${ }^{1}$ Gastrointestinal Surgery, ${ }^{2}$ Translational Oncology and ${ }^{3}$ Specialized Surgeries, Graduate School of Medical \\ and Dental Sciences, Tokyo Medical and Dental University, Bunkyo-ku, Tokyo 113-8519, Japan
}

Received February 11, 2019; Accepted October 7, 2019

DOI: $10.3892 /$ ol.2019.11091

\begin{abstract}
The present study investigated aberrant methylation in colorectal cancer (CRC) and its impact on characteristics and prognosis of patients with CRC. Bone morphogenetic protein 2 (BMP2) was identified as a target gene in oligonucleotide microarray expression profiling in a previous study. Subsequently, the BMP2 methylation status was assessed in 498 patients with stage I-III CRC using methylation-specific polymerase chain reaction, and the association between $B M P 2$ methylation status, patient characteristics and prognosis was assessed. BMP2 methylation was observed in 302/498 (60.6\%) patients and was associated with positive lymph nodes and venous invasion $(\mathrm{P}<0.05)$. In the stage III subgroup, overall survival (OS) was significantly worse in the methylated $B M P 2$ group compared with in the unmethylated $B M P 2$ group $(\mathrm{P}=0.012) . B M P 2$ methylation was identified as an independent factor for poor OS in stage III patients $(\mathrm{P}=0.041)$. Notably, in the left-sided stage III CRC subgroup, relapse-free survival and OS were significantly worse in the methylated $B M P 2$ group than in the unmethylated group $(\mathrm{P}=0.048$ and $\mathrm{P}=0.031$, respectively). In conclusion, DNA hypermethylation of $B M P 2$ was a poor prognostic factor in patients with stage III disease, particularly in those with left-sided stage III CRC. BMP2 methylation may be a biomarker for prognosis prediction and treatment decision-making.
\end{abstract}

Correspondence to: Dr Toshiaki Ishikawa, Department of Specialized Surgeries, Graduate School of Medical and Dental Sciences, Tokyo Medical and Dental University, 1-5-45 Yushima, Bunkyo-ku, Tokyo 113-8519, Japan

E-mail: ishi.srg2@tmd.ac.jp

Key words: colorectal cancer, bone morphogenetic protein 2, methylation, prognostic factor, methylation-specific polymerase chain reaction, tumor-suppressor gene

\section{Introduction}

Colorectal cancer (CRC) is the third most commonly diagnosed malignancy and the fourth leading cause of cancer-related death globally (1). The T (tumor), $\mathrm{N}$ (node), and M (metastasis) factors of the 'TNM classification of malignant tumors' published by the Union for International Cancer Control (UICC) have been accepted as robust predictors of the prognosis of cancer patients and provide the basis for decision-making in CRC treatment strategies (2-4). In contrast, for a more precise stratification of CRC treatment, various prognostic and/or predictive factors have been studied $(5,6)$.

Aberrant methylation of a gene promoter $\mathrm{CpG}$ island is an epigenetic change that silences gene expression and is a crucial mechanism that inactivates tumor-suppressor genes and promotes cancer progression (7). The present study focused on DNA methylation and searched for clinically significant tumor-suppressor genes in CRC by screening for candidate genes suspected to be silenced by DNA methylation using microarray analysis. Bone morphogenetic protein 2 (BMP2) was identified as the candidate gene.

BMP2 was detected as a bone morphogenetic factor with activity for inducing bone morphogenesis (8). It belongs to the transforming growth factor (TGF)- $\beta$ superfamily and plays important roles in generation, cell differentiation, proliferation, and apoptosis (9-11). In CRC, BMP2 has been reported as a tumor-suppressor gene (12). However, the relationship between $B M P 2$ and clinicopathological factors has not been studied in clinical CRC cases.

The present study aimed to investigate the relationship between DNA methylation of $B M P 2$ and clinicopathological factors and prognosis of patients with CRC.

\section{Materials and methods}

Identification of the target gene by microarray gene expression analysis. In the current study, the microarray data was used from a previous study (13). The gene expression data are deposited in the Gene Expression Omnibus (http://www.ncbi.nlm.nih. gov/geo/) under accession number GSE32323.

Probe sets from cell lines were selected according to the following criteria: i) FC $>2.0$ compared with that of the 
CpG island methylator phenotype (CIMP) RKO cell line and ii) up-regulation of gene expression in at least two CRC cell lines. For the paired clinical samples, probe sets were selected for $\mathrm{FC}$ of normal versus tumor tissue $(\mathrm{N} / \mathrm{T})>1.5$ (i.e., higher expression in normal tissue than in tumor tissue) (14), and 99 genes (123 probes) that appeared to be suppressed by DNA methylation were identified (Table SI). We examined the published literature and narrowed down candidate genes in the context of genes that are hypermethylated in neoplasms, but the clinical significance of inactivation remained unclear in CRC. Finally, we selected $B M P 2$ as the target gene of interest (Fig. 1).

Cell lines. Seven CRC cell lines (RKO, SW480, HT29, HCT116, COLO201, LoVo, and DLD1) were obtained from the American Type Culture Collection. These cell lines were maintained in Dulbecco's modified Eagle's medium or RPMI1640 medium (Gibco; Thermo Fisher Scientific, Inc.) containing $10 \%$ heat-inactivated fetal bovine serum, 100 units $/ \mathrm{ml}$ of penicillin, $100 \mu \mathrm{g} / \mathrm{ml}$ of streptomycin, $10 \mathrm{mM}$ of HEPES, and $1.0 \mathrm{mM}$ of sodium pyruvate and were incubated at $37^{\circ} \mathrm{C}$ in $5 \% \mathrm{CO}_{2}$. Cultured cells were pelletized and used to isolate total genomic DNA for methylation assay and total RNA for mRNA expression assay.

Patients. This study included primary tumors from 498 patients (290 male and 208 female patients) who underwent curative surgical resection for CRC at Tokyo Medical and Dental University Hospital between 2008 and 2013. Of these 498 patients, 91 had stage I disease, 204 had stage II disease, and 203 had stage III disease. The median patient age was 69.0 years (range, 29-93 years). Patients did not receive any treatment prior to surgery. Postoperative adjuvant chemotherapy was administered to 14 patients with stage II disease (6.9\%) and 150 patients with stage III disease (73.9\%). The median follow-up period at analysis was 63 months (range, 0-122 months). Samples were included in the methylation assay.

Methylation assay. We used methylation-specific polymerase chain reaction (MSP) to evaluate the methylation status of BMP2 (15). The phenol/chloroform method was used to isolate total genomic DNA from cell lines and surgically resected tumor samples. Bisulfite treatment was performed using the EpiTect Plus DNA Bisulfite kit (Qiagen), according to the manufacturer's instructions. Bisulfite-modified DNA was then used as template DNA for polymerase chain reaction (PCR) amplification with PCR primers corresponding to the region affected by methylation. The methylation-specific and unmethylation-specific primer sequences of $B M P 2$ were based on the results of the study by Wen et al (16). MSP was performed using the EpiTect MSP kit (Qiagen). The PCR conditions of iCycler ${ }^{\mathrm{TM}}$ (Bio-Rad Laboratories Inc.) were as follows: $95^{\circ} \mathrm{C}$ for $10 \mathrm{~min} ; 40$ cycles at $94^{\circ} \mathrm{C}$ for $15 \mathrm{sec}, 62^{\circ} \mathrm{C}$ for $30 \mathrm{sec}$, and $72^{\circ} \mathrm{C}$ for $30 \mathrm{sec}$ (methylated) or 40 cycles at $94^{\circ} \mathrm{C}$ for $15 \mathrm{sec}, 58^{\circ} \mathrm{C}$ for $30 \mathrm{sec}$, and $72^{\circ} \mathrm{C}$ for $30 \mathrm{sec}$ (unmethylated); and finally $72^{\circ} \mathrm{C}$ for $10 \mathrm{~min}$. EpiTect control DNA (Qiagen) was used as a positive control. After amplification, electrophoresis of PCR products was performed using $2.5 \%$ agarose gels. When positive amplification was noted with methylation-specific primers, irrespective of whether amplification was detected with unmethylation-specific primers, the tumor was considered as a 'methylated' tumor. When no amplification was noted with methylation-specific primers, the tumor was considered as an 'unmethylated' tumor.

mRNA expression assay. The mRNA expression level of $B M P 2$ in each cell line was assessed using reverse transcription-quantitative PCR (RT-qPCR). Total RNA was extracted using the RNeasy mini kit (Qiagen). cDNA was synthesized using the High Capacity cDNA Reverse Transcription kit (Applied Biosystems; Thermo Fisher Scientific, Inc.), according to the manufacturer's instructions, with iCycler ${ }^{\mathrm{TM}}$ (Bio-Rad Laboratories, Inc.). cDNA was then amplified by PCR using a fluorescence-based real-time detection method with the ABI Prism 7300 real-time PCR system (Applied Biosystems; Thermo Fisher Scientific, Inc.), according to the manufacturer's protocol. TaqMan $B M P 2$ and $\beta$-actin (ACTB) Gene Expression Assay-on-demand (BMP2 Assay ID, Hs00154192_m1; ACTB Assay ID, Hs99999903_m1; Applied Biosystems; Thermo Fisher Scientific, Inc.) were used for RT-qPCR. The mRNA expression level of $B M P 2$ in each sample was normalized to that of ACTB (internal standard). The relative quantification of $B M P 2$ mRNA expression was performed by the $\Delta \Delta \mathrm{Cq}$ method using SDS v1.4 with RQv1.0 software (Applied Biosystems; Thermo Fisher Scientific, Inc.) (17). Each analysis was performed in triplicate.

Statistical analysis. The chi-square test was used to estimate differences between the groups. Relapse-free survival (RFS) was calculated from the date of surgery to recurrence or death, whichever occurred first. Overall survival (OS) was calculated from the date of surgery to death from any cause. RFS and OS curves were estimated using the Kaplan-Meier method, and log-rank tests were used to assess treatment differences overall. Factors affecting RFS and OS were examined with univariate and multivariate analyses using the Cox proportional hazards model, and Cox models were used to estimate hazard ratios (HRs) and their confidence intervals (CIs). All statistical analyses were performed using EZR (Saitama Medical Center, Jichi Medical University, Saitama, Japan), which is a graphical user interface for R (The R Foundation for Statistical Computing, Vienna, Austria). More precisely, it is a modified version of $\mathrm{R}$ commander designed to add statistical functions frequently used in biostatistics (18). $\mathrm{P}<0.05$ was considered to indicate a statistically significant difference.

Ethical considerations. This study was conducted in accordance with the Declaration of Helsinki and its later amendments or comparable ethical standards. The study protocol was approved by the Institutional Review Board of Tokyo Medical and Dental University, and written informed consent was obtained from all patients before enrollment.

\section{Results}

Identification of BMP2 as a target gene by microarray gene expression analysis. As shown in Fig. 1, the microarray data was used from a previous study (13). We defined 'candidate genes' according to the following criteria: i) FC $>2.0$ compared with that of the CIMP RKO cell line and ii) up-regulation of 


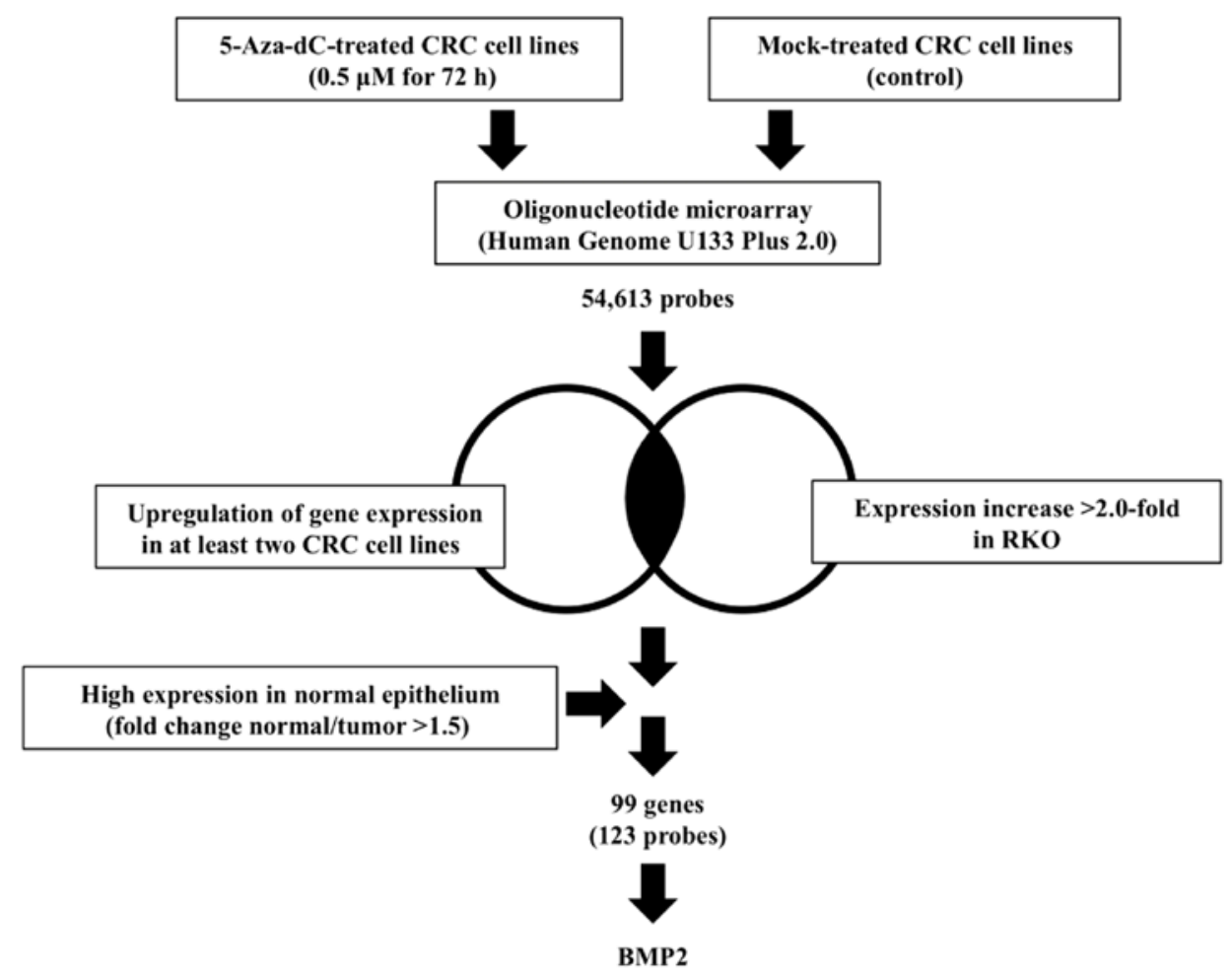

Figure 1. Outline of selection of candidate genes in CRC. 5-Aza-DC, 5-aza-2-deoxycytidine; CRC, colorectal cancer; BMP2, bone morphogenetic protein 2.

gene expression in at least two CRC cell lines. For the paired clinical samples, probe sets were selected for FC of normal versus tumor tissue $>1.5$. 99 candidate genes were identified among the 54,613 probes (Table SI). We examined the published literature for additional analysis of these genes. In order to identify epigenetically affected genes with methylation, we first excluded genes in neoplasm not affected by methylation. In addition, we removed genes with high or unknown expression in neoplasm, genes with hypomethylation or unknown methylation status in neoplasm. We selected $B M P 2$ reported to be a tumor-suppressor gene in CRC and DNA aberrant hypermethylation in neoplasms, but the clinical significance remain unclear as a target gene for further investigation.

BMP2 methylation and $m R N A$ expression in cell lines. MSP was performed in seven CRC cell lines, and BMP2 methylation was detected in the following three cell lines: RKO, HCT116, and COLO201 (Fig. 2A). With regard to mRNA expression detected by RT-qPCR, the expressions in these three cell lines with $B M P 2$ methylation was quite low compared with the expressions in the other four cell lines without $B M P 2$ methylation (Fig. 2B), suggesting that the mRNA expression of $B M P 2$ was down-regulated by DNA hypermethylation.

BMP2 methylation and patient characteristics. The relationships between $B M P 2$ methylation status and important clinicopathological factors are shown in Table I. BMP2 methylation was observed in 302 of the 498 patients $(60.6 \%)$. $B M P 2$ methylation was associated with positive lymph nodes $(\mathrm{P}=0.012)$, venous invasion $(\mathrm{P}=0.027)$, and stage III disease $(\mathrm{P}=0.010)$. There were no associations of $B M P 2$ methylation with sex, tumor location, histological type, tumor invasion depth, and lymphatic invasion.
BMP2 methylation and prognosis of patients with $C R C$. The RFS and OS curves of all 498 patients are presented in Fig. 3. The 5-year RFS rates in the methylated BMP2 and unmethylated $B M P 2$ groups were 70.0 and $75.5 \%$, respectively (Fig. 3A). The 5-year OS rates in the methylated BMP2 and unmethylated BMP2 groups were 82.8 and $90.3 \%$, respectively (Fig. 3B). OS tended to be worse in the methylated $B M P 2$ group than in the unmethylated $B M P 2$ group (RFS, $\mathrm{P}=0.142$; OS, $\mathrm{P}=0.067$ ).

The RFS and OS curves stratified by TNM-stage are presented in Fig. 4. In the stage I and II subgroups, there were no differences in both RFS and OS between the methylated $B M P 2$ and unmethylated $B M P 2$ groups (Fig. 4A-D). On the other hand, in the stage III subgroup, OS was significantly worse and RFS was marginally worse in the methylated BMP2 group than in the unmethylated $B M P 2$ group $(\mathrm{P}=0.012$ and $\mathrm{P}=0.061$, respectively) (Fig. 4E and F).

BMP2 methylation and prognosis of stage III patients. We focused on stage III patients and investigated the impact of BMP2 methylation status on prognosis (Table II). With regard to poor RFS, in the univariate analysis, left-sided colon $(\mathrm{P}=0.004)$, high tumor invasion depth $(\mathrm{P}=0.022)$, and lymph node metastasis $(\mathrm{P}=0.003)$ were identified as risk factors. In the multivariate analysis involving variables with $\mathrm{P}$-values $<0.10$ in the univariate analysis, high age $(\mathrm{HR}=1.67 ; 95 \%$ CI, 1.05-2.64; $\mathrm{P}=0.029)$, left-sided colon $(\mathrm{HR}=2.28 ; 95 \% \mathrm{CI}$, 1.34-3.87; $\mathrm{P}=0.002)$, high tumor invasion depth $(\mathrm{HR}=1.73$; 95\% CI, 1.08-2.77; $\mathrm{P}=0.022)$, and lymph node metastasis $(\mathrm{HR}=1.75 ; 95 \% \mathrm{CI}, 1.10-2.79 ; \mathrm{P}=0.019)$ were identified as independent factors for poor RFS. With regard to poor OS, in the univariate analysis, high age $(\mathrm{P}<0.001)$, lymph node metastasis $(\mathrm{P}=0.026)$, and $B M P 2$ methylation $(\mathrm{P}=0.016)$ were risk factors. In the multivariate analysis, high age $(\mathrm{HR}=2.97 ; 95 \%$ 
A
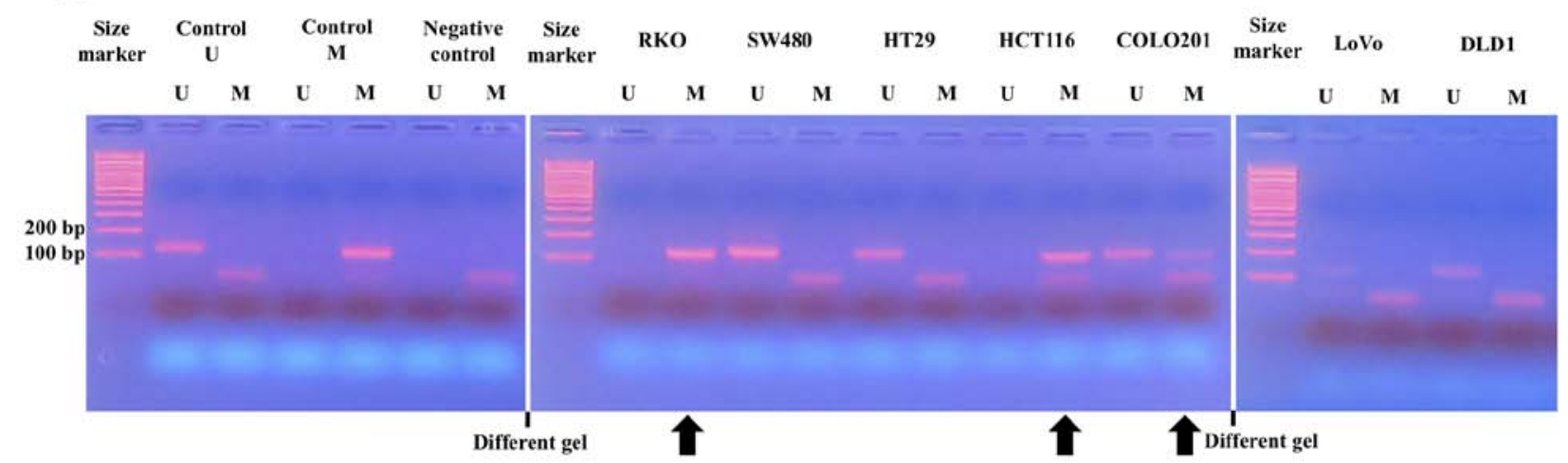

B

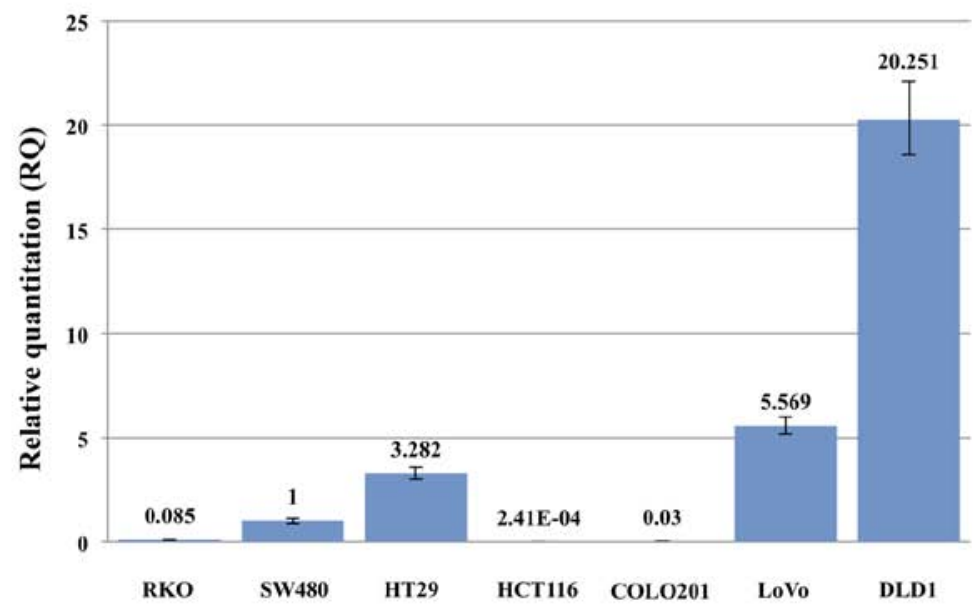

Figure 2. Methylation and mRNA expression levels of $B M P 2$ in cell lines. (A) Methylation-specific polymerase chain reaction analysis of $B M P 2$ in seven CRC cell lines. RKO, HCT116 and COLO201 cells exhibited a BMP2 methylation band. Non-specific bands that are considered to be primer dimers are shown in smaller size than the $B M P 2$ methylation band. Different gels are separated by white lines. (B) RT-qPCR analysis of $B M P 2 \mathrm{mRNA}$ expression in seven CRC cell lines. RKO, HCT116, and COLO201 cells, which were demonstrated to exhibit methylation in (A), had lower mRNA expression than that in the other cell lines. $B M P 2$, bone morphogenetic protein 2; CRC, colorectal cancer; RT-qPCR, quantitative reverse transcription-quantitative polymerase chain reaction; $\mathrm{U}$, unmethylated; $\mathrm{M}$, methylated; RQ, relative quantification.

CI, 1.58-5.59; $\mathrm{P}<0.001)$, lymph node metastasis $(\mathrm{HR}=1.96 ; 95 \%$ CI, 1.05-3.66; $\mathrm{P}=0.035)$, and $B M P 2$ methylation $(\mathrm{HR}=2.36$; 95\% CI, 1.04-5.39; $\mathrm{P}=0.041)$ were identified as independent factors for poor OS.

As the prognostic impact of sidedness has attracted attention in recent years, we investigated the relationship between $B M P 2$ methylation status and prognosis according to the sidedness of the primary tumor (19). In right-sided colon cancer patients, there were no differences in both RFS and OS between the methylated and unmethylated $B M P 2$ groups (Fig. 5A and B). On the other hand, in left-sided colon cancer patients, RFS and OS were significantly worse in the methylated $B M P 2$ group than in the unmethylated group (RFS, $\mathrm{P}=0.048$; OS, $\mathrm{P}=0.031$; Fig. 5C and D).

\section{Discussion}

To our knowledge, this is the first report to demonstrate that BMP2 methylation affects the clinical outcomes of CRC patients. Among 498 study patients with curatively resected stage I-III CRC, BMP2 methylation was observed in $60 \%$, and it was more common in patients with lymph node metastasis and venous invasion. In addition, patients with $B M P 2$ methylation and stage III disease, especially left-sided CRC, had a poor prognosis.
BMP2, a member of the TGF- $\beta$ superfamily, exerts its effect via two types of transmembrane serine/threonine kinase receptors [BMP receptor type I (BMPRI) and II (BMPRII)]. BMP2 induces the recapitulation of endochondral bone formation when appropriate undifferentiated cells are exposed to it. Additionally, BMPs, including BMP2, are found in many tissues, and they perform physiological functions (20). When BMP2 binds to BMPRII, BMPRI is phosphorylated and the downstream signaling is activated via the Sma- and Mad-related (Smad) protein (Smad1/5/8). Phosphorylated Smad1/5/8 subsequently forms complexes with Smad4, translocates to the nucleus, and finally regulates transcription in cooperation with transcriptional factors $(11,21)$. Through this Smad signaling pathway, BMP2 stimulates $\mathrm{p} 21^{\mathrm{CIP} 1 / \mathrm{WAF} 1}$, a cell cycle inhibitor that blocks the activity of cyclin-dependent kinase (CDK). Inhibition of CDK suppresses the phosphorylation of $\mathrm{Rb}$, a transcriptional regulator, and then causes cell cycle arrest at the G1 or G0 phase (11). BMP2 has been also reported to induce apoptosis; however, the details of the mechanism are still unclear $(12,22)$. Based on these findings, $B M P 2$ was reported as a tumor-suppressor gene in various cancers, including $\mathrm{CRC}$, and its down-regulation was suggested to be involved in cancer progression (10,12,22-25). Furthermore, in several malignancies, down-regulation of 
Table I. BMP2 methylation and patients characteristics.

\begin{tabular}{|c|c|c|c|c|}
\hline Variables & Met, n (n=302) & Unm, n $(n=196)$ & Rate of $B M P 2-$ Met, $\%$ & P-value \\
\hline \multicolumn{5}{|l|}{ Age at surgery, years } \\
\hline$\leq 70$ & 161 & 123 & 56.7 & \multirow[t]{2}{*}{0.047} \\
\hline$\geq 71$ & 141 & 73 & 65.9 & \\
\hline \multicolumn{5}{|l|}{ Sex } \\
\hline Male & 182 & 108 & 62.8 & \multirow[t]{2}{*}{0.294} \\
\hline Female & 120 & 88 & 57.7 & \\
\hline \multicolumn{5}{|l|}{ Tumor location } \\
\hline Right-sided colon & 102 & 63 & 61.8 & \multirow[t]{3}{*}{0.123} \\
\hline Left-sided colon & 77 & 66 & 53.8 & \\
\hline Rectum & 123 & 67 & 64.7 & \\
\hline \multicolumn{5}{|l|}{ Histological type } \\
\hline G1 (pap, tub1) & 98 & 49 & 66.7 & \multirow[t]{3}{*}{0.203} \\
\hline $\mathrm{G} 2(\mathrm{tub} 2)$ & 180 & 129 & 58.3 & \\
\hline G3 (por1, por2, muc, sig) & 24 & 18 & 57.1 & \\
\hline \multicolumn{5}{|l|}{ Tumor invasion depth } \\
\hline $\mathrm{T} 1$ & 26 & 18 & 59.1 & \multirow[t]{4}{*}{0.827} \\
\hline $\mathrm{T} 2$ & 49 & 26 & 65.3 & \\
\hline $\mathrm{T} 3$ & 167 & 110 & 60.3 & \\
\hline $\mathrm{T} 4$ & 60 & 42 & 58.8 & \\
\hline \multicolumn{5}{|l|}{ LN metastasis } \\
\hline No & 163 & 132 & 55.3 & \multirow[t]{3}{*}{0.012} \\
\hline N1 & 99 & 46 & 68.3 & \\
\hline $\mathrm{N} 2$ & 40 & 18 & 69.0 & \\
\hline \multicolumn{5}{|l|}{ Lymphatic invasion } \\
\hline Absent & $163^{\mathrm{a}}$ & 119 & 57.8 & \multirow[t]{2}{*}{0.177} \\
\hline Present & 138 & 77 & 64.2 & \\
\hline \multicolumn{5}{|l|}{ Venous invasion } \\
\hline Absent & $47^{\mathrm{a}}$ & 47 & 50.0 & \multirow[t]{2}{*}{0.027} \\
\hline Present & 254 & 149 & 63.0 & \\
\hline \multicolumn{5}{|l|}{ TNM 7th stage } \\
\hline I & 53 & 38 & 58.2 & \multirow[t]{3}{*}{0.010} \\
\hline II & 110 & 94 & 53.9 & \\
\hline III & 139 & 64 & 68.5 & \\
\hline
\end{tabular}

${ }^{\mathrm{a}} 1$ unknown. Right-sided colon included cecum, ascending and transverse colon. Left-sided colon included descending and sigmoid colon. Met, methylated; Unm, unmethylated; BMP2, bone morphogenetic protein 2; LN, lymph node; pap, papillary adenocarcinoma; tub, tubular adenocarcinoma; por, poorly differentiated adenocarcinoma; muc, mucinous adenocarcinoma; sig, signet-ring cell carcinoma.

$B M P 2$ was reported to be caused by DNA promoter methylation $(16,26)$. In CRC, DNA aberrant hypermethylation in the promoter region of $B M P 2$ has been reported previously (27). In the present study, BMP2 methylation was observed in $60 \%$ of patients, suggesting that it played an important role in cancer progression.

Du et al (26) reported that BMP2 methylation was related to chemoresistance in breast cancer patients. Additionally, Mitsui et al (22) reported that BMP2 methylation had an impact on the prognosis of patients with renal cell carcinoma. With regard to CRC, a correlation between BMP2 and chemoresistance was previously reported in in vitro studies $(25,27)$.
However, no report has assessed the impact of $B M P 2$ methylation on clinical outcomes in CRC patients.

There are some possible reasons why the outcomes in the methylated $B M P 2$ group were poor among stage III patients. First, the efficacy of adjuvant chemotherapy might be low in patients with $B M P 2$ methylation. As mentioned above, a correlation between $B M P 2$ methylation and chemoresistance has been presumed in previous reports $(25,26)$. In our exploratory analysis using a small subset of stage III patients with adjuvant chemotherapy, the methylated BMP2 group $(n=97)$ had a higher recurrence rate and worse OS when compared with the findings in the unmethylated group $(n=53)$ (recurrence rate, 
$\mathbf{A}$

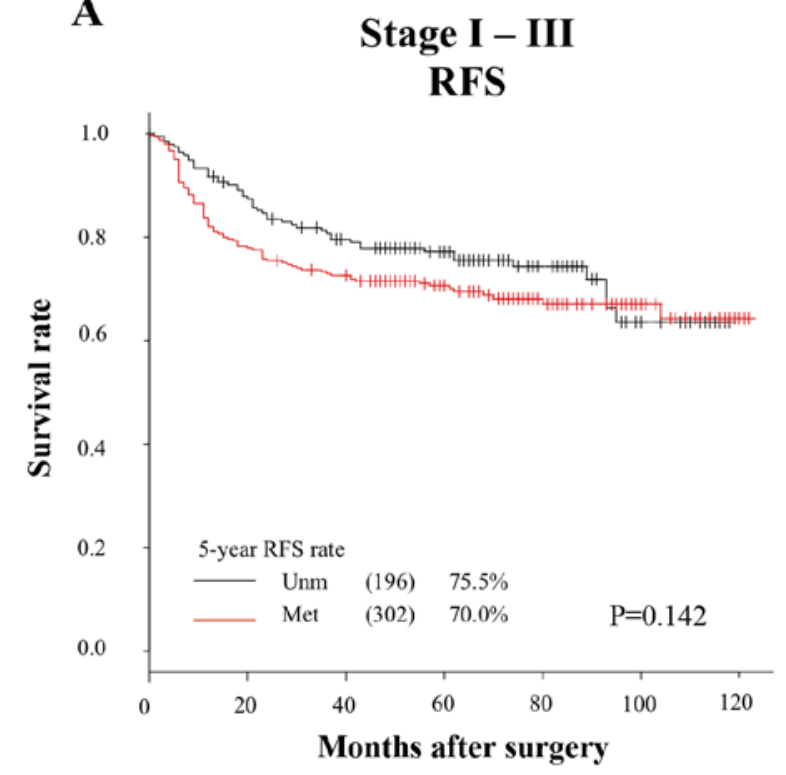

B

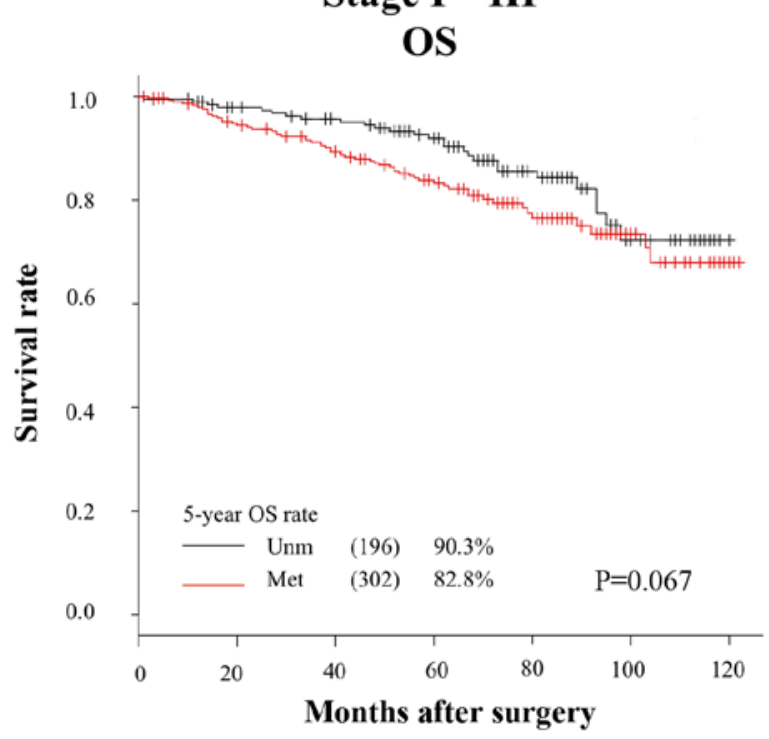

Figure 3. RFS and OS according to the BMP2 methylation status. Patients with stage I, II and III CRC (n=498) were divided into methylated and unmethylated BMP2 groups, and (A) RFS and (B) OS were assessed using Kaplan-Meier curves. OS tended to be worse in the methylated BMP2 group than in the unmethylated $B M P 2$ group. Unm, unmethylated; Met, methylated; RFS, relapse-free survival; OS, overall survival; $B M P 2$, bone morphogenetic protein 2; $\mathrm{CRC}$, colorectal cancer.
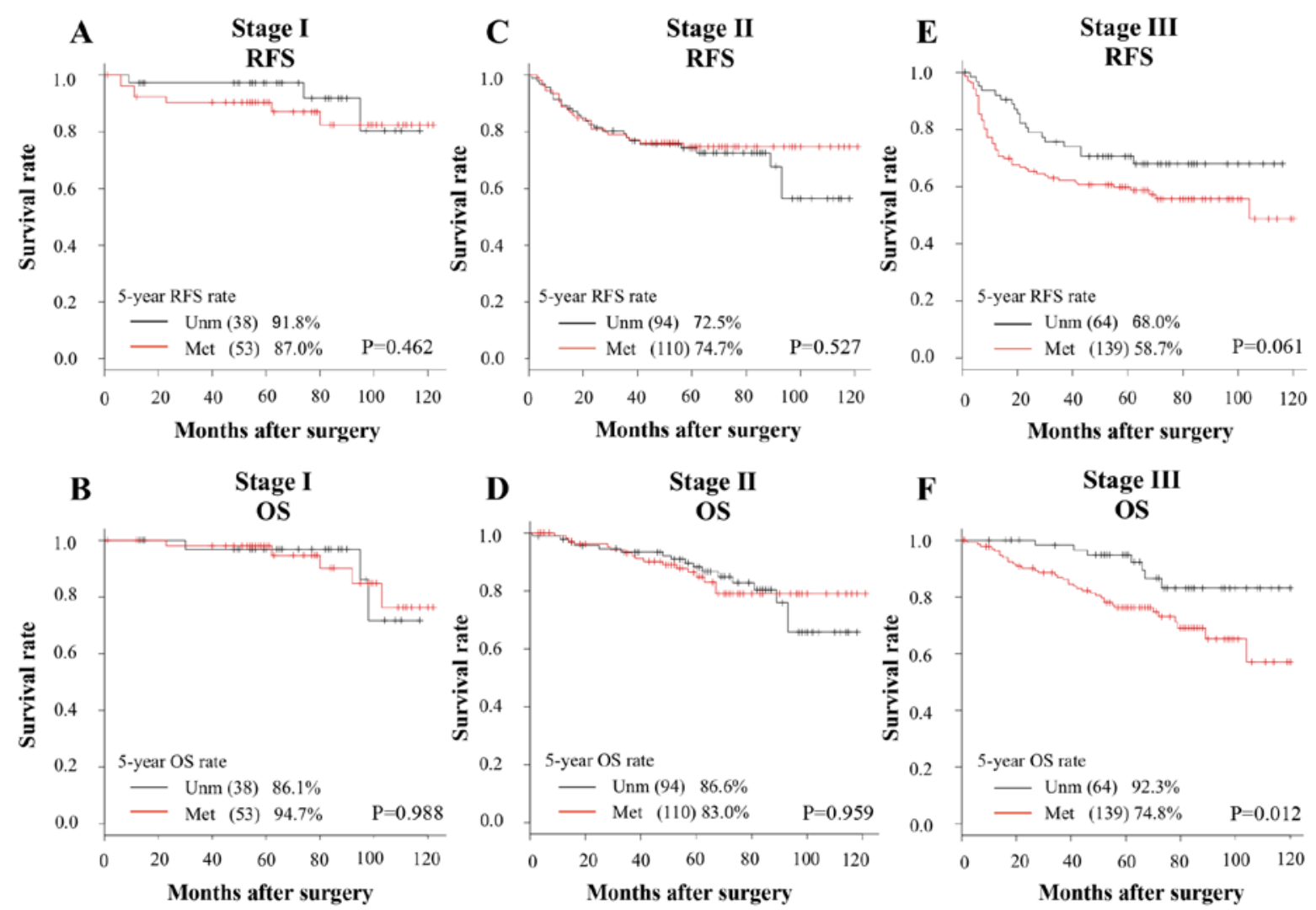

Figure 4. RFS and OS according to the $B M P 2$ methylation status and disease stage. (A) RFS and (B) OS in stage I patients (n=91). (C) RFS and (D) OS in stage II patients $(\mathrm{n}=204)$. There were no differences identified between both RFS and OS in the methylated $B M P 2$ and unmethylated $B M P 2$ groups. (E) RFS and (F) OS in stage III patients $(\mathrm{n}=203)$. OS was significantly worse and RFS was marginally worse in the methylated $B M P 2$ group compared with in the unmethylated $B M P 2$ group. Unm, unmethylated; Met, methylated; RFS, relapse-free survival; OS, overall survival; $B M P 2$, bone morphogenetic protein 2.

$38.1 \%$ vs. $26.4 \%$; OS, $\mathrm{P}=0.039)$. However, there was no difference in survival between the methylated and unmethylated $B M P 2$ groups among patients without adjuvant chemotherapy (data not shown). Second, clinical outcomes after recurrence might be related to the methylation status of $B M P 2$. In our series of stage III patients, survival time after recurrence was 


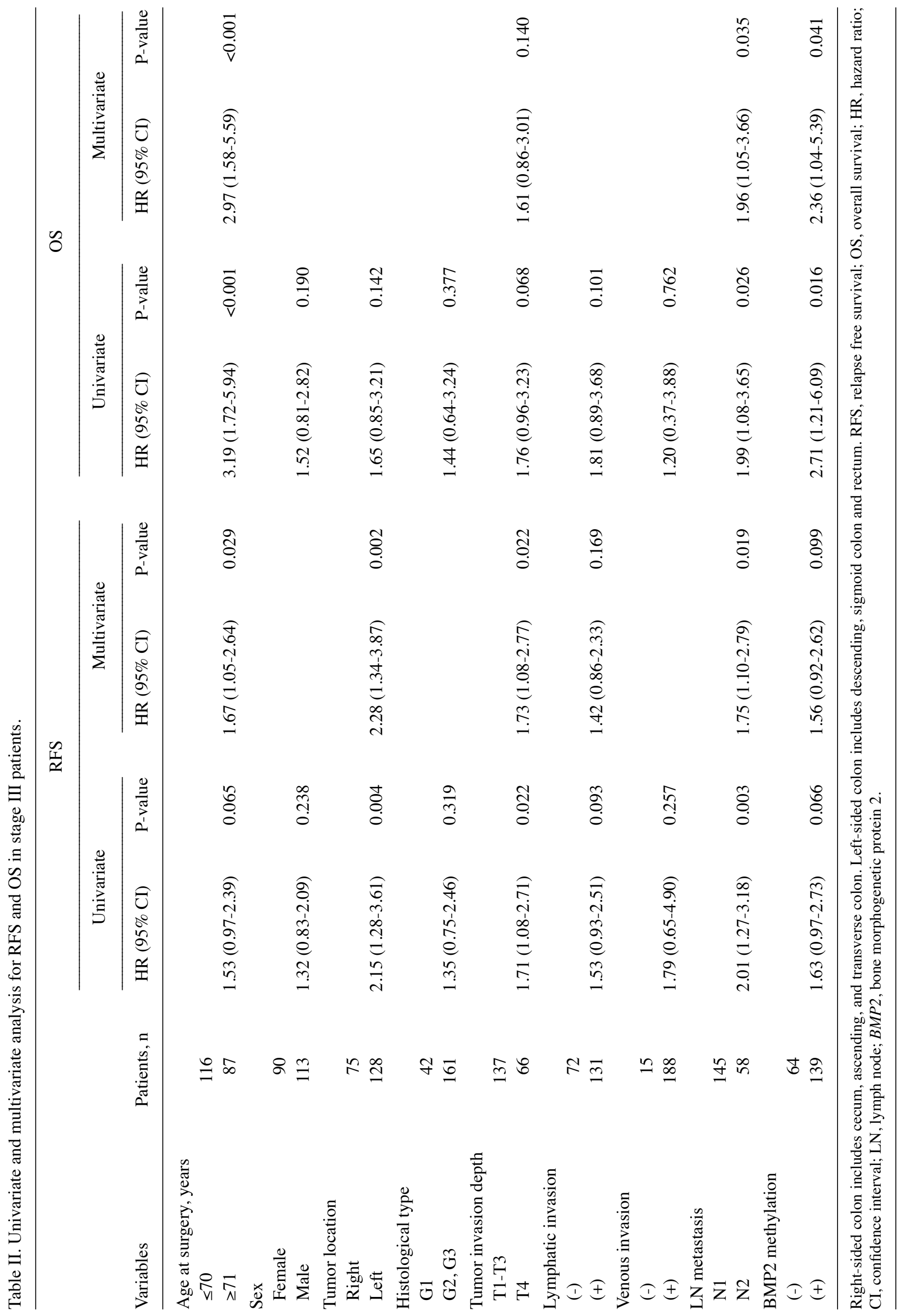



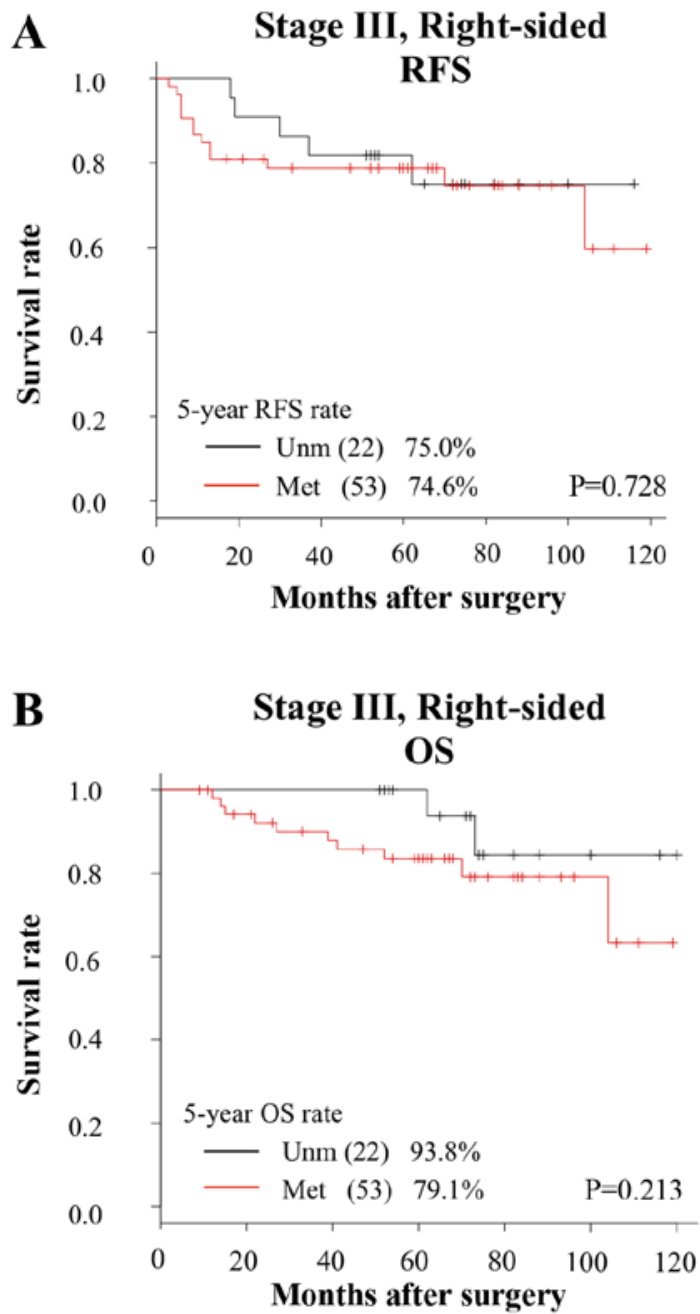

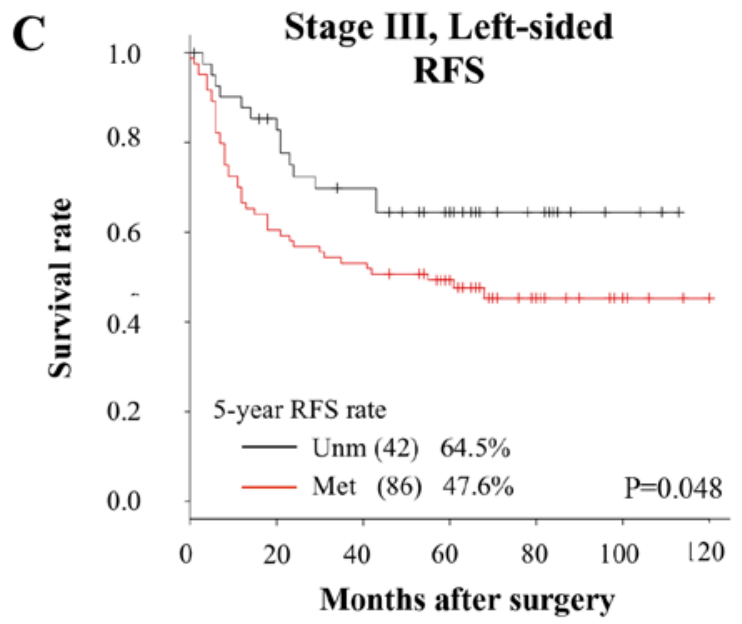

D

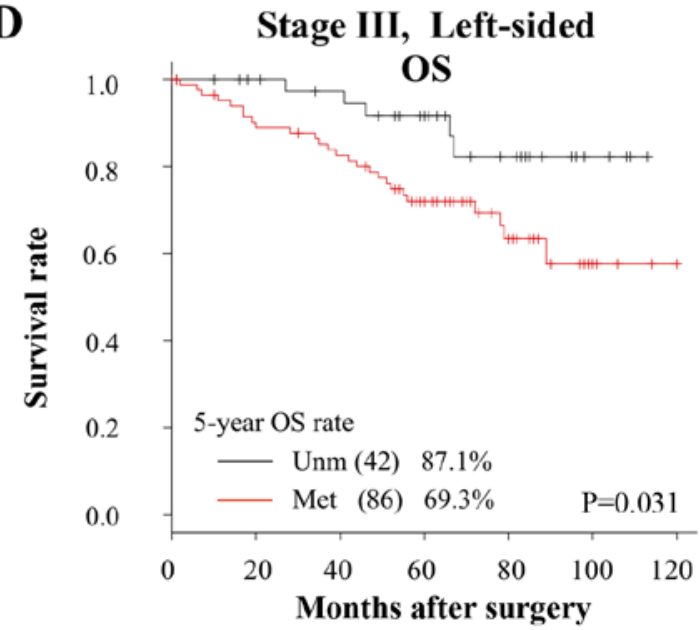

Figure 5. RFS and OS according to the $B M P 2$ methylation status and location in stage III patients. (A) RFS and (B) OS in right-sided patients (n=75). There were no differences identified between both RFS and OS in the methylated and unmethylated BMP2 groups. (C) RFS and (D) OS in left-sided patients (n=128). RFS and OS were significantly worse in the methylated $B M P 2$ group than in the unmethylated group. Unm, unmethylated; Met, methylated; RFS, relapse-free survival; OS, overall survival; $B M P 2$, bone morphogenetic protein 2.

significantly shorter in the methylated $B M P 2$ group $(n=53)$ than in the unmethylated group $(n=18)$, and the median survival times were 26 and 44 months in the methylated and unmethylated $B M P 2$ groups, respectively $(\mathrm{P}=0.033$, data not shown). As there was no difference in the site of recurrence such as liver, lung and others, or resection rate after recurrence, it is presumed that there was a difference in the effect of chemotherapy after recurrence between the two groups. A further study on the impact of $B M P 2$ methylation using a larger cohort is required.

Recently, it has been reported that the molecular profile and biological characteristics of CRC can vary according to the sidedness of the tumor, and the primary tumor location is one of the promising prognostic factors (19). In our study, with a focus on the sidedness in stage III disease, RFS and OS were significantly worse in the methylated $B M P 2$ group than in the unmethylated $B M P 2$ group among stage III patients with left-sided CRC. One possible reason for these findings might be the correlation between the $B M P 2$ signaling pathway and molecular characteristics of left-sided CRC. Smad4 loss and p53 have been reported to be more common in left-sided colon cancer (28-30). In cases of Smad4 loss and/or p53 mutation, the expression of $\mathrm{p} 21^{\mathrm{CIP} 1 / \mathrm{WAF} 1}$ is presumed to be suppressed (31). Therefore, when a tumor has BMP2 methylation and Smad4 loss/p53 mutation, cell cycle regulation by $\mathrm{p} 21^{\mathrm{CIP} 1 / \mathrm{WAF} 1} \mathrm{might}$ reduce, which could be an advantage for cancer progression.

We have some future perspectives for the clinical use of $B M P 2$ methylation. First, BMP2 methylation might be useful for the pre-treatment prediction of lymph node metastasis. Our results indicated that $B M P 2$ methylation in the primary tumor was associated with lymph node metastasis. If lymph node metastasis can be predicted with absolute accuracy by checking BMP2 methylation in biopsy specimens or endoscopically resected $\mathrm{T} 1$ tumors, it will be quite useful for deciding whether radical surgery with lymph node dissection should be recommended, which will have a great clinical impact. In our small series of surgically resected T1 cases with a positive lymph node $(\mathrm{n}=12), B M P 2$ methylation of primary tumor tissue was observed in 11 cases (91.7\%) (data not shown). For the clinical use of the $B M P 2$ methylation status in lymph node metastasis prediction, future studies involving a large number of $\mathrm{T} 1$ cancer cases and a prospective cohort are needed. Second, $B M P 2$ methylation, as a risk factor for recurrence and short survival, might be useful for decision 
making with regard to the treatment strategy. It might be better to follow CRC patients with $B M P 2$ methylation very closely, as $B M P 2$ methylation is a poor prognostic factor. Furthermore, the development of a $B M P 2$ demethylating agent in the future might help in CRC treatment.

The present study had several limitations. First, we cannot exclude the possibility of bias owing to the retrospective and single institutional selection of the study population. Further multicenter collaborative prospective studies are required to confirm the results of the present study. Second, the number of $\mathrm{CpG}$ sites that we analyzed was small. Future methylation studies for BMP2 in CRC are required to confirm our results. Third, we did not assess the methylation status of $B M P 2$ in normal colon cell lines.

In conclusion, DNA hypermethylation of $B M P 2$ is a poor prognostic factor in patients with stage III disease, especially those with left-sided stage III CRC. BMP2 methylation might be a biomarker for prognosis prediction and treatment decision-making; however, further studies are needed.

\section{Acknowledgements}

The authors thank Mrs. Yoko Takagi and Mrs. Junko Inoue (Department of Specialized Surgeries, Graduate School of Medical and Dental Sciences, Tokyo Medical and Dental University, Bunkyo-ku, Tokyo 113-8519, Japan) for their excellent technical assistance.

\section{Funding}

No funding was received.

\section{Availability of data and materials}

All data generated or analyzed during the present study are included in this published article. The datasets generated and/or analyzed during the current study are available in the Gene Expression Omnibus repository (https://www.ncbi.nlm. nih.gov/geo/query/acc.cgi?acc=GSE32323).

\section{Authors' contributions}

TYM, TI, MI, SY, TM and HU were involved in the conception and design of the study, and development of the methodology. TYM performed the experiments and collected clinicopathological data. TYM, TI and MI analyzed the results. TYM, TI, MI and SO edited the manuscript. TI, MI, SO, HB, AK, SY, TMa, HU and YK assisted with all assays and analyses and in manuscript preparation. TI, MI, SO, HB, AK, SY, TM, HU and YK supervised the study. All authors have read and approved the final manuscript.

\section{Ethics approval and consent to participate}

The present study was conducted in accordance with the Declaration of Helsinki and its later amendments or comparable ethical standards. The study protocol was approved by the Institutional Review Board of Tokyo Medical and Dental University, and written informed consent was obtained from all patients before enrollment.

\section{Patient consent for publication}

Not applicable.

\section{Competing interests}

The authors declare that they have no competing interests.

\section{References}

1. Arnold M, Sierra MS, Laversanne M, Soerjomataram I, Jemal A and Bray F: Global patterns and trends in colorectal cancer incidence and mortality. Gut 66: 683-691, 2017.

2. National Comprehensive Cancer Network: Clinical Practice Guidelines in Oncology; Colon cancer version 2.2018. https://www.nccn.org/professionals/physician_gls/pdf/colon.pdf. Accessed May 2, 2018.

3. Labianca R, Nordlinger B, Beretta GD, Mosconi S, Mandalà M, Cervantes A and Arnold D; ESMO Guidelines Working Group: Early colon cancer: ESMO clinical practice guidelines for diagnosis, treatment and follow-up. Ann Oncol 24: vi64-vi72, 2013.

4. Watanabe T, Muro K, Ajioka Y, Hashiguchi Y, Ito Y, Saito Y, Hamaguchi T, Ishida H, Ishiguro M, Ishihara S, et al: Japanese Society for Cancer of the Colon and Rectum (JSCCR) guidelines 2016 for the treatment of colorectal cancer. Int J Clin Oncol 23: 1-34, 2018.

5. Erstad DJ, Tumusiime G and Cusack JC Jr: Prognostic and predictive biomarkers in colorectal cancer: Implications for the clinical surgeon. Ann Surg Oncol 22: 3433-3450, 2015.

6. Guinney J, Dienstmann R, Wang X, de Reyniès A, Schlicker A, Soneson C, Marisa L, Roepman P, Nyamundanda G, Angelino P, et al: The consensus molecular subtypes of colorectal cancer. Nat Med 21: 1350-1356, 2015.

7. Kalari S and Pfeifer GP: Identification of driver and passenger DNA methylation in cancer by epigenomic analysis. Adv Genet 70: 277-308, 2010.

8. Wozney JM, Rosen V, Celeste AJ, Mitsock LM, Whitters MJ, Kriz RW, Hewick RM and Wang EA: Novel regulators of bone formation: molecular clones and activities. Science 242 : 1528-1534, 1988.

9. Hardwick JC, Van Den Brink GR, Bleuming SA, Ballester I, Van Den Brande JM, Keller JJ, Offerhaus GJ, Van Deventer SJ and Peppelenbosch MP: Bone morphogenetic protein 2 is expressed by, and acts upon, mature epithelial cells in the colon. Gastroenterology 126: 111-121, 2004.

10. Zhang J, Ge Y, Sun L, Cao J, Wu Q, Guo L and Wang Z: Effect of bone morphogenetic protein-2 on proliferation and apoptosis of gastric cancer cells. Int J Med Sci 9: 184-192, 2012.

11. Davis H, Raja E, Miyazono K, Tsubakihara Y and Moustakas A: Mechanisms of action of bone morphogenetic proteins in cancer. Cytokine Growth Factor Rev 27: 81-92, 2016.

12. Zhang Y, Chen X, Qiao M, Zhang BQ, Wang N, Zhang Z, Liao Z, Zeng L, Deng Y, Deng F, et al: Bone morphogenetic protein 2 inhibits the proliferation and growth of human colorectal cancer cells. Oncol Rep 32: 1013-1020, 2014.

13. Khamas A, Ishikawa T, Shimokawa K, Mogushi K, Iida S, Ishiguro M, Mizushima H, Tanaka H, Uetake H and Sugihara K: Screening for epigenetically masked genes in colorectal cancer Using 5-Aza-2'-deoxycytidine, microarray and gene expression profile. Cancer Genomics Proteomics 9: 67-75, 2012.

14. Iwata N, Ishikawa T, Okazaki S, Mogushi K, Baba H, Ishiguro M, Kobayashi H, Tanaka H, Kawano T, Sugihara K and Uetake H: Clinical significance of methylation and reduced expression of the quaking gene in colorectal cancer. Anticancer Res 37: 489-498, 2017.

15. Herman JG, Graff JR, Myöhänen S, Nelkin BD and Baylin SB: Methylation-specific PCR: A novel PCR assay for methylation status of CpG islands. Proc Natl Acad Sci USA 93: 9821-9826, 1996.

16. Wen XZ, Akiyama Y, Baylin SB and Yuasa Y: Frequent epigenetic silencing of the bone morphogenetic protein 2 gene through methylation in gastric carcinomas. Oncogene 25: 2666-2673, 2006.

17. Livak KJ and Schmittgen TD: Analysis of relative gene expression data using real-time quantitative PCR and the 2(-Delta Delta C(T)) method. Methods 25: 402-408, 2001.

18. Kanda Y: Investigation of the freely available easy-to-use software 'EZR' for medical statistics. Bone Marrow Transplant 48: 452-458, 2013 . 
19. Lee GH, Malietzis G, Askari A, Bernardo D, Al-Hassi HO and Clark SK: Is right-sided colon cancer different to left-sided colorectal cancer? -a systematic review. Eur J Surg Oncol 41: 300-308, 2015.

20. Tian H, Zhao J, Brochmann EJ, Wang JC and Murray SS: Bone morphogenetic protein-2 and tumor growth: Diverse effects and possibilities for therapy. Cytokine Growth Factor Rev 34: 73-91, 2017.

21. Miyazono K, Kamiya Y and Morikawa M: Bone morphogenetic protein receptors and signal transduction. J Biochem 147: 35-51, 2010.

22. Mitsui Y, Hirata H, Arichi N, Hiraki M, Yasumoto H, Chang I, Fukuhara S, Yamamura S, Shahryari V,Deng G, et al: Inactivation of bone morphogenetic protein 2 may predict clinical outcome and poor overall survival for renal cell carcinoma through epigenetic pathways. Oncotarget 6: 9577-9591, 2015.

23. Brubaker KD, Corey E, Brown LG and Vessella RL: Bone morphogenetic protein signaling in prostate cancer cell lines. J Cell Biochem 91: 151-160, 2004.

24. Johnsen IK, Kappler R, Auernhammer CJ and Beuschlein F: Bone morphogenetic proteins 2 and 5 are down-regulated in adrenocortical carcinoma and modulate adrenal cell proliferation and steroidogenesis. Cancer Res 69: 5784-5792, 2009.

25. Vishnubalaji R, Yue S, Alfayez M, Kassem M, Liu FF, Aldahmash A and Alajez NM: Bone morphogenetic protein 2 (BMP2) induces growth suppression and enhances chemosensitivity of human colon cancer cells. Cancer Cell Int 16: 77, 2016.

26. Du M, Su XM, Zhang T and Xing YJ: Aberrant promoter DNA methylation inhibits bone morphogenetic protein 2 expression and contributes to drug resistance in breast cancer. Mol Med Rep 10: 1051-1055, 2014.
27. Kodach LL, Jacobs RJ, Voorneveld PW, Wildenberg ME, Verspaget HW, van Wezel T, Morreau H, Hommes DW, Peppelenbosch MP, van den Brink GR and Hardwick JC: Statins augment the chemosensitivity of colorectal cancer cells inducing epigenetic reprogramming and reducing colorectal cancer cell 'stemness' via the bone morphogenetic protein pathway. Gut 60: 1544-1553, 2011

28. Shen H, Yang J, Huang Q, Jiang MJ, Tan YN, Fu JF, Zhu LZ, Fang XF and Yuan Y: Different treatment strategies and molecular features between right-sided and left-sided colon cancers. World J Gastroenterol 21: 6470-6478, 2015.

29. Natsume S, Yamaguchi T, Takao M, Iijima T, Wakaume R, Takahashi K, Matsumoto H, Nakano D, Horiguchi SI, Koizumi K and Miyaki M: Clinicopathological and molecular differences between right-sided and left-sided colorectal cancer in Japanese patients. Jpn J Clin Oncol 48: 609-618, 2018

30. Russo A, Bazan V, Iacopetta B, Kerr D, Soussi T and Gebbia N; TP53-CRC Collaborative Study Group: The TP53 colorectal cancer international collaborative study on the prognostic and predictive significance of p53 mutation: Influence of tumor site, type of mutation, and adjuvant treatment. J Clin Oncol 23: 7518-7528, 2005.

31. Kastenhuber ER and Lowe SW: Putting p53 in context. Cell 170: 1062-1078, 2017.

This work is licensed under a Creative Commons Attribution-NonCommercial-NoDerivatives 4.0 International (CC BY-NC-ND 4.0) License. 